\title{
Towards Understanding the Adoption and Social Experience of Digital Wallet Systems
}

\author{
Shiliang Tang ${ }^{1 \dagger}$, Ziming $\mathrm{Wu}^{2 \dagger}$, Xinyi Zhang ${ }^{1}$, Gang Wang ${ }^{3}$, Xiaojuan $\mathrm{Ma}^{2}$, Haitao Zheng ${ }^{4}$, Ben Y. Zhao ${ }^{4}$ \\ UC Santa Barbara ${ }^{1}$, Hong Kong University of Science and Technology ${ }^{2}$, Virginia Tech ${ }^{3}$, University of Chicago ${ }^{4}$ \\ \{shiliang_tang, xyzhang\}@cs.ucsb.edu,zwual@connect.ust.hk, gangwang@vt.edu, \\ mxj@cse.ust.hk, \{htzheng, ravenben\}@cs.uchicago.edu \\ †equal contribution
}

\begin{abstract}
For millions around the globe, digital wallets are replacing cash and credit cards. These services support user-to-user payments, and add a social component to transactions. However, there is little understanding of the key factors behind digital wallets' rapid growth in US (Venmo) and China (WeChat Pay). What are the factors that led to their success? How social relationships play a role in their adoption? We conduct a mixed methods study, using a comprehensive survey $(N=879)$ and semi-structured interviews $(N=41)$ to explore the interplay of the two roles of these digital wallets, i.e., a payment system and a social platform. Our analysis suggests that the network effect does benefit their adoption and retention, but through different mechanisms. In return, transaction activities performed in digital wallets help strengthen existing social ties. We also present design implications for future social payment services.
\end{abstract}

\section{Introduction}

Today's Internet has dramatically reshaped the way in which people make payments and transfer money. The biggest paradigm shift is the emergence of online and mobile payment services, which have started to replace cash and/or credit cards around the globe. For example, PayPal, which began as an offshoot of eBay in 1998, has built up a 210 million user base by 2017. Alipay, PayPal's counterpart in China reached 450 million users in 2017.

More recently, Venmo (USA) and WeChat Pay (China) entered the market as digital wallet services that target peer-to-peer (P2P) payments, where users send and receive money from each other digitally. These new services quickly gained significant market shares, surprising many in the finance industry with their rapid growth. WeChat Pay has reached 200 million users in only three years, and Venmo (acquired by Paypal in 2013) has attracted 10 million active users within the same time span. Compared to conventional online payment systems, the most distinctive feature that distinguishes $\mathrm{P} 2 \mathrm{P}$ digital wallets is the integration of established social networks to facilitate payments between friends.

There have been many attempts to connect social features with financial transactions, by leveraging the potential power of social networks in attracting and retaining users. The results have been mixed at best. For example, Facebook has integrated P2P payments into Facebook Messenger [1]; Snapchat also offers a money transfer feature in their messaging app [2]; and Alipay is experimenting with a new social network service over its already successful e-commerce system [3].

Other than Venmo and WeChat Pay, most attempts to integrate social links into financial payments have met limited success. Questions then arise: what are the universal and culture-specific factors that contribute to the rapid adoption of Venmo and WeChat Pay? What roles do the social network and financial interactions play in this process? Many prior works on focus on transactions between strangers [4, 5]. Nevertheless, transactions via peer-to-peer digital wallets often involve parties that already knows each other, i.e., friends, which leads to different behaviors. For example, Venmo users tend to create unambiguous messages when sending money to strangers but would write funny and clever transaction descriptions to friends [6]. In this paper, we aim to discover answers to the above questions by taking social factors into consideration, which can shed light on mobile software adaption for the research community and industrial practitioners.

We conduct a mixed-method study that consists of an online survey (380 Venmo users in the US and 499 WeChat Pay users in China), and an in-depth interview (21 US Venmo users and 20 Chinese WeChat Pay users). From the survey and interview results, we obtain key insights into why and how users adopt a P2P digital 
wallet, users' perceptions, behaviors, and experiences when using the services, and their projection of the future directions moving forward.

\section{Background}

In recent years, digital wallet systems such as Venmo (US) and WeChat Pay (China) are undertaking tremendous growth. Known for their user-to-user payments and social components, Venmo and WeChat Pay have taken significant market shares from traditional online payment systems (e.g., PayPal and Alipay), forcing them to make changes.

Venmo. Venmo is a digital wallet app with 11 million users as of May 2016. First launched in 2009, Venmo has been growing tremendously. In a single year of 2015, Venmo increased its transaction volume by $200 \%$, and reached 12 billion dollars in quarterly transaction volume in the first quarter of 2018 [7, 8]. On Venmo, users can transfer money to each other and build social links. Users can either pay or charge another user for some given amount, and attach a short message (e.g., "my rent"). A unique feature of Venmo is social sharing. For each transaction, users can choose to share the transaction record to the "public" (default) or with "friends." The transaction information, excluding the actual amount, will be visible in the public stream or their friends' news feed. Other users can like or comment on shared transactions. Finally, users can use the app to pay for online/offline services (e.g., restaurants) that have registered Venmo accounts.

WeChat Pay. WeChat Pay is another fast-growing social digital wallet. It has gained 600 million users in only four years since its launch in 2013 [9]. WeChat Pay is built into WeChat, the largest social messaging network in China, which has more than 900 million monthly active users [9]. Similar to Venmo, WeChat Pay allows users to transfer money with friends and pay for various online or offline services from e-commerce sites to hospitals and taxis. Usually, a user starts a transaction by posting a message in another user's chatbox or scan the user's payment QR code. Unlike Venmo, WeChat Pay does not support social sharing of transaction records. Instead, WeChat Pay has a unique feature called "Moment" that supports sharing feature within a limited social scope. Besides, it develops a feature called "Red Packet" which mimics the Chinese tradition that people give red envelopes (with cash in it) to friends and family members for best wishes. This WeChat Pay feature allows users to send digital red envelopes to friends or use the money to start a lottery draw among a group of friends (each gets a random amount). During the Chinese New Year of 2018, 688 million users sent and/or received red envelopes through WeChat Pay [10].

\section{Theoretical Background and Related Work}

\subsection{Social Factors Drive Technology Adoption}

One of our key goals is to understand how P2P digital wallets become so widely-adopted in a short period of time, especially how social aspects contribute to the adoption process. Theories in diffusion and adoption of technology may provide possible insights into this question.

Diffusion of Innovation (DOI) theory [11] postulates the detailed mechanisms of how a newly innovated technology spreads across populations. It identifies four elements of diffusion: (1) the innovation itself, (2) channels through which it is communicated, (3) over time, and (4) among members of a social system. Designs related to each of these elements may speed up or slow down the diffusion process. In this paper, we are particularly interested in the role that the integration of social functions into digital wallets may play in this process.

Literatures on social science and marketing have shown that an online social network (element 2 in DOI) can impact users' decision-making process, as exemplified by the word-of-mouth [12] and herding phenomena [13]. Zsolt et al. further suggested that social network can exert two key effects on people's decisions to take up a technology: degree effect and cluster effect [14]. The former indicates that individuals are inclined to follow the decisions of their connected friends, while the latter suggests that people are influenced by the majority decision of others around them. Especially when encountering uncertainty of new technologies, users would always take their social network as informative and trustworthy referents [15].

The involvement of members of an established social system (element 4 in DOI) may have additional benefits for the spread of P2P digital wallets. For one thing, public sharing in the social system can serve as a mass media for information and opinion dissemination [11]. For another, interpersonal messaging in the social system is an effective means of persuasion [11 16]. Both communication channels have the potential to increase user acceptance of $\mathrm{P} 2 \mathrm{P}$ digital wallets.

In this paper, we are interested in verifying whether the role of social systems played in the spread of Venmo and WeChat Pay services is consistent with the DOI theory. Previously, Wang et al. found that perceived social influence and networking ability of 
WeChat Pay contribute to its adoption [17]. Zhang et $a l$. quantitatively showed that Venmo's social network structure is denser than traditional social networks [18]. Compared to prior works, this paper aims to use the Diffusion of Innovation (DOI) theory as a lens to gain a deep understanding of (1) how social features in a digital wallet affect adoption of digital wallets, and (2) how the perceptions and mechanisms vary in different cultural contexts. We hypothesize that the adoption of Venmo and WeChat Pay propagates through their internal social network by a mixed effect of degree effect and clustering effect (H1).

\subsection{Social Interactions Impact Financial Systems}

According to a generic model of trust in e-commerce settings [19], for a user to join a transaction, their level of trust needs to exceed perceived risk. On the one hand, in the face of high levels of uncertainty and opportunism, people tend to conduct financial activities (e.g., mobile banking [20]) with social ties of greater trust and confidence in seek of some security, as explained in the Social Exchange Theory (SET) [21]. On the other hand, interpersonal interactions during a transaction help users build and fortify bonds with their trading partners [4], which ultimately strengthen the trust relationships [22, 23]. More recently, Caraway et al. showed that social awareness stream in Venmo provides users with the opportunities for familiarizing with the app and keeping up with friends, sometimes at the cost of privacy and comfort [6].

To service providers' interest, customers' social connections also affect their retention behavior [24]. It has been suggested that pre-existing social ties within a group [25], lightweight socialization and successful early social experience [26, 27] may prevent members from leaving an (online) community. In a recent qualitative study, Gui et al. showed that interactions over pre-existing social network boost the retention rate of fitness-tracking applications [28].

In this paper, other than studying how relationship affects transactional behaviors, we are interested in examining if social relationships have an effect on user retention using Social Exchange Theory, especially from the perspective of trust building. We hypothesize that social relationships between users help build trust and increase user engagement of Venmo and WeChat Pay (H2).

\subsection{Social Function of Currency}

Aside from being a depersonalized and asocial means of exchange [4, 29], money can serve as a medium that bears social and cultural meanings [30]. In other words, money can shape how people behave in social settings, and its usage can be affected by social relationships. Such effect varies under different culture contexts.

In recent years, the emergence of electronic payment/exchange services in different forms has provoked discussions about the socio-cultural role of money and other alternative and complementary currencies in new contexts. There have been competing views on how electronic payment systems can affect social relationships. On the one hand, prior research suggested that cashless exchange processes may undermine social sensitivity and increase social isolation [22, 31, 32]. For example, Pritchard et al. showed that cashless practice of London buses reduced the potential interaction between drivers and passengers compared to cash payment [33]. On the other hand, alternative payment systems can benefit social relationships in various ways. Ferreira et al. examined how the slow and cumbersome procedure of cashless payment system stimulates interpersonal connections [4]. Mainwaring et al. showed how the design of e-cash in Japan ties to Japanese moral virtue of smooth flow and avoidance of commotion [34]. In some cases, electronic payments are used as means to show care for friends and families at a distance [35].

Venmo and WeChat Pay, representatives of emerging digital wallet services, intend to expedite peer-to-peer money-based payment while promoting social interactions among users, which seem to be two conflicting goals according to the aforementioned findings. In this paper, we aim at studying whether (and how) the new paradigm of $\mathrm{p} 2 \mathrm{p}$ digital wallets succeeds in supporting easy and positive social experiences with clear social benefits for targeted users, a recommendation for peer systems [36], despite its utilitarian commitment. We hypothesize that transaction experiences brought by Venmo and WeChat Pay bring positive value to user social connections (H3).

\section{Research Methodology}

To test our hypothesizes, we conduct mixed methods research to explore user perceptions and behaviors in P2P digital wallets. Mixed methods research is a methodology widely used in social and behavioral studies [37, 38]. It involves collecting, analyzing and integrating close-ended quantitative (e.g., experiments and surveys) and open-ended qualitative (e.g., focus groups and interviews) research data. In this way, we can offset weaknesses inherent to using a single type of research activity, triangulate findings, and extend the 
breadth and depth of insights [6, 39]. More specifically, we first conducted a large-scale survey on both Venmo and WeChat Pay users to understand their usage of mobile digital wallet. Then, based on the survey results, we conducted interviews to further obtain more details about their usage scenarios, and their perception about social relationship and financial activities.

\subsection{Survey}

We conducted surveys to understand the success of mobile digital wallets, exploring the underlying role of social functions. We framed our questions around the components of Diffusion of Innovation theory: how digital wallet is used and propagates through social network. Our survey contains four main sections: First, we asked users about their digital wallet usage including how they got started, their usage frequency and usage scenarios. Second, we focused on the social features to understand how the users perceive the value of social transactions and sharing. Third, we asked about users' perspective on different aspects of making a better digital wallet in the future. Finally, we collected demographic information. We deployed the same survey for both Venmo users (in English) and WeChat Pay users (translated to Chinese). For each survey, we used multiple channels to obtain a more diverse population.

Our Venmo survey was hosted on SurveyMonkey1. We recruited users by directly contacting Venmo users via the Venmo app (220 participants), and through Amazon Mechanical Turk (160 participants).

- Venmo-Direct: We first recruited participants by directly contacting Venmo users. We got in touch with users by making a one-cent transaction to them on Venmo and attached a short request for participation in our survey. As compensation, we paid each participant who completed the survey $\$ 1$ and added them to a random drawing for $\$ 300$. In total, we randomly sent 2,381 requests and received 220 valid responses, giving us a response rate of $9.24 \%$. This is a reasonable response considering Venmo is not a typical survey platform, and some of the sampled users may no longer be using Venmo.

- Venmo-MTurk: We augmented our Venmo user population by crowd-sourcing on Amazon Mechanical Turk (MTurk)2. We confirmed that each crowdworker is indeed a Venmo user through their ID. We paid each worker $\$ 0.5$ via MTurk and paid another $\$ 0.5$ through their Venmo account (for account verification). We received submissions from

\footnotetext{
${ }^{1}$ http://www . surveymonkey.com/

${ }^{2}$ https://www.mturk.com/
}

176 MTurk workers, from which we removed 16 $(9.1 \%)$ responses from workers who registered their Venmo accounts after our HIT had been published.

Our WeChat Pay survey was hosted on the survey platform WenJuanXing 3, a Chinese counterpart to SurveyMonkey. We advertised our survey on social media accounts (209 participants) and made use of WenJuanXing's own user recruitment service (290 participants). Again, respondents who didn't use WeChat Pay were filtered out during analysis.

- WeChat-Social Media: Since there was no way to randomly contact WeChat Pay users, we advertised our survey requests on our social media accounts (e.g., WeChat and Weibo) to invite our friends to participate and spread the survey. In total, we collected 217 valid responses. We identified and removed 8 users who have never used WeChat Pay, leaving us with 209 valid responses.

- WeChat-Recruit: We also recruited participants by leveraging WenJuanXing's own user recruitment service. Although WenJuanXing could not explicitly target WeChat Pay users, the wide adoption of WeChat Pay in China means this was an efficient way to reach WeChat Pay users. In total, 290 out of the 300 purchased responses were from valid WeChat Pay users.

It is worth noting that we recruit users from two difference channels for surveys in each country. To test whether it is reasonable to combine surveys responses from two recruitment methods, we broke down the pools and examined results for all the survey questions. All of our statements are consistent between samples from different recruiting methods, so all following analysis will be based on a combination of responses from two recruitment methods.

The demographics of respondents are listed below. Among all 380 Venmo survey participants, 52.9\% were female, $45.8 \%$ were male, and $1.3 \%$ chose "Others". The majority of participants $(67.4 \%)$ were between age 21 to $30,15.0 \%$ were younger than $21,4.5 \%$ were older than 40, and the rest fell between 31 to 40 . For WeChat survey participants, $42.5 \%$ were female and $57.5 \%$ were male. Similarly, the majority of users $(49.5 \%)$ were between age 21 to $30,9.4 \%$ were younger than 21 , $16.2 \%$ were older than 40 , and the rest fell between 31 to 40 .

\subsection{Interview}

Finally, we conducted in-depth interviews to understand user experience on digital wallet usage,

${ }^{3}$ https://www. sojump.com/ 
perceptions on the social networks in digital wallets, and their opinions on financial activities. Our interview is semi-structured, primarily to explore users' personal experiences with Venmo or WeChat Pay.

We recruited participants by advertising publicly through our social media accounts and via word-of-mouth. In total, 21 Venmo users from the US and 20 WeChat Pay users from China participated in the interviews conducted by two of the authors, either face-to-face or over Skype. We find the sample size to be sufficient since few new topics emerged in the last few participants of the interview [40].

Each user took part in two interview sessions with a total length of 45 to 60 minutes, first discussing their perception and expectation of $\mathrm{P} 2 \mathrm{P}$ digital wallets in general and then sharing their experiences with social interactions in these services. We took audio recordings of each interview session (with user consent). After conducting all interviews, two of the authors transcribed and conducted a thematic analysis of the interview responses using open coding. A third author audited the coding process and helped resolve different opinions.

The demographics of our interview participants roughly match the demographics of our survey participants. In China, 11 out of the 20 participants were female, with $80 \%$ aged from 21 to $30,5 \%$ below 20 , and $15 \%$ above 30 . In US, we had 13 female interviewees out of 21 , with $71 \%$ between the age of 21 and $30,10 \%$ below 21 years old, and 19\% above 30 .

\section{Adoption and Usage of Digital Wallets}

Our first question is why Venmo and WeChat Pay get popular so quickly. We find that both degree effect and cluster effect in Diffusion Of Innovation theory play a role in the adoption of $\mathrm{P} 2 \mathrm{P}$ digital wallets $(\mathrm{H} 1)$, but their effects are different for Venmo and WeChat Pay.

\subsection{Degree Effect Contributes to Venmo's Adoption}

For Venmo users, "friends" is the most voted reason for starting to use the digital wallet (79.2\%, Figure 1 . As shown in Figure 2] "making transactions with friends" is also the most common usage scenario voted by Venmo users (93\%). In particular, 37\% of users chose "making transactions with friends" as their only usage case of Venmo, indicating the importance of social network in their wallet usage.

We further explore how social influence affects adoption in the interviews. The result is straightforward: many Venmo users accepted the app because of direct recommendations from their friends (US except 2, 8, 9, $14,15)$.
A bunch of us have gone on a trip, so we wanted to split expenses. A few of them were already using Venmo, so they were just like "oh, there is this app you can download, and it's super easy to send money to each other." (US10, female, age 21-30)

In other words, the adoption in Venmo largely fits the description of degree effect, i.e., individuals are inclined to follow the decision of their connected friends.

\subsection{Cluster Effect Contributes to WeChat Pay's Adoption}

Unlike for Venmo, our survey results show that WeChat Pay users do not feel strongly that they embrace the service because of their friends' usage. However, in the interviews, we find that social effect does exist in the adoption process of WeChat Pay, but in a more implicit manner. It hides largely behind a cultural feature called "red envelope".

To be more specific, in our survey, more WeChat Pay users stated that their primary intents to use the service were "easy to use" and "avoid carrying cash", rather than "friends" (Figure 1). This seems to suggest a less powerful network effect on the initial adoption of WeChat Pay. We also find that, although "making transactions with friends" is one of the most common activities on WeChat Pay (Figure 2), only $8 \%$ of users put it as the sole usage scenario. This may be because in addition to P2P transactions WeChat Pay supports many Customer-to-Business payment activities, which relate less to users' social network.

However, when we try to verify this finding in the interview, we discovered that WeChat Pay also benefits greatly from the social network effect, through its "Red Packet" feature. It turns out that most people became aware of the payment system in WeChat because they received Red Packets from others or got involved in Red Packet grabbing activities in WeChat groups (CN2, 3, 4, $7,8,9,10,11,13,15,20)$.

At the beginning, lots of my friends sent red envelopes in WeChat groups to celebrate Spring Festival. It looked interesting and entertaining which got me attracted to participating in those activities. But it required me to bind a bank card to WeChat Pay. I did so. And after that, I found it convenient to use WeChat Pay to pay and transfer money. (CN3 female, age 21-30)

Red envelope activities in this sense raised user awareness of its payment function, lowering the entry barriers. This phenomenon fits the description of cluster effect, i.e., users start using a technology under the 


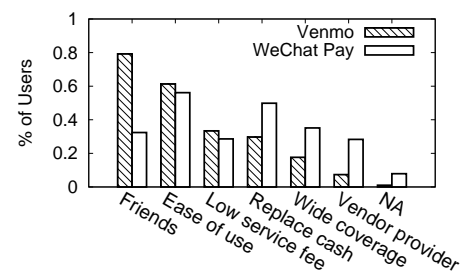

Figure 1. Reasons for starting to use digital wallets.

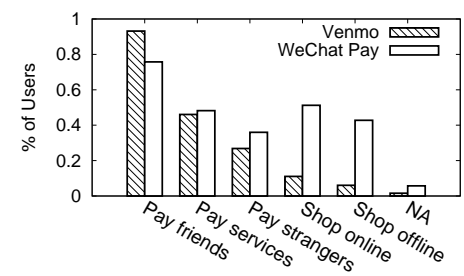

Figure 2. Common usage scenarios.

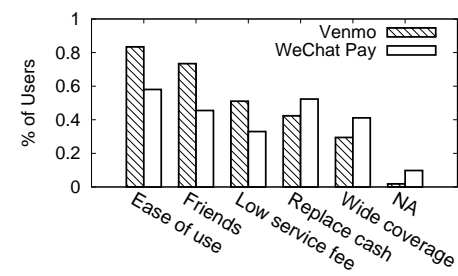

Figure 3. Reasons for continuing to use digital wallets. influence of the majority decision of others around. WeChat Pay users built a sense of "community usage" when the Red Packet became a national fad during the Chinese New Year.

Overall, we reveal that the social features help to accelerate the adoption of both Venmo and WeChat Pay, but the underlying mechanisms are slightly different for the US and Chinese users. More specifically, Venmo users build their confidence in the system based on actions and opinions of their close friends (strong ties), while the Chinese users are likely to be influenced by the community (Red Packet usage in various discussion groups).

\section{Social Ties Affect Transaction Experience}

Besides initial adoption, we find that social relationships also impact user interactions with and through the digital wallets in several aspects. In general, social relationships help build trust and increase user retention $(\mathrm{H} 2)$, but the user experiences can be different between different types of social relationships.

\subsection{Social Relationship Builds Trust During Transactions}

According to a generic model of trust in e-commerce settings [19], for users to join a transaction, their level of trust needs to exceed the perceived risk.

In the context of digital wallets, first, social relationships help users overcome security concerns in digital wallets and establish trust in the control mechanism. Our interviews show that some users hesitated to use digital wallets at first (US1, 17, 20, 21, $\mathrm{CN} 3,4,5,7,8,9,12,15)$. People worried that digital wallets make money transfer too easy (US12, 13, 17). Chinese users (CN1, 5, 7, 9, 15, 18) mentioned that WeChat Pay gives them a sense of "insecurity" because it grows out of an open social network service. It is the trust on other digital wallet users, e.g., faith in the friend who recommended the service or confidence built upon the app's large user base, that turned apprehension into acceptance (US1, 3, 7, 10, 13, 14, 17, 19, 21, CN 11, 13).

I trusted it [Venmo] because the person who suggested it to me is very knowledgeable about computer security, so I trusted his opinion. (US7, female, age 31-40)

Second, trust developed in offline social relationships can be transferred online, adding an additional layer of protection against potential risks. People felt that even if an online payment was unsuccessful, they could still find the associated friend offline and fix the transaction (US5, 7, 21).

I don't need to worry about how to recall the money back. I can just knock on his door. If I know this person well, I don't really worry about Venmo's safety issue. (US21, male, age 21-30)

\subsection{Social Ties Affect Retention}

"Friends" is one of the most important reasons for Venmo users to keep the app (Figure 3). Those who reported "making transactions with friends" to be the sole purpose of Venmo claimed that they would definitely leave the service if their friends stopped using it (US except 2, 5, 11, 18).

It's just like any other social network. If more people are on one thing than another, then I will just end up switching. I just use whatever people are using. Because you have to connect them, rather than convert them. (US9, female, age 31-40)

However, social ties do not seem to play as significant a role in WeChat Pay as they do in Venmo. Convenience seems to be a stronger incentive for WeChat Pay users to stay engaged. They put "easy to use" and "replacing cash" over "friends" as the top two reasons for retaining the service (Figure 3). On the one hand, $90 \%$ of the Chinese respondents claimed that they will still use WeChat Pay even if some friends 
drop the service. It is because WeChat Pay provides other convenient payment functions that they use quite frequently, e.g., paying phone bills, calling taxi, etc. $(\mathrm{CN} 2,3,4,6,14)$.

On the other hand, one of the WeChat Pay users indicated that if a considerable number of people stop using the service round them or even across the country, she would consider terminating it, thinking that there are some problems with the system (CN17).

In a word, user feedback suggests that degree effect occurs in the retention of Venmo users, while user engagement in WeChat Pay exhibits sings of cluster effect, which is similar to the findings about adoption.

\subsection{Types of Social Relationships Impact Transactional Experiences}

Although social ties bring trust to the digital service, they may experience social awkwardness during transactions. We hypothesize that social intimacy affects the ease with which users undertake transactions. In our interview, we define three groups of people based on their social distance to the interviewees, i.e., close friends, normal friends and acquaintances, from strong to weak ties. For each of our participants, we asked them about their transaction experience with the three groups respectively. We focused on how their perceived level of comfort varies with the strength of each social tie. We find that people feel different levels of comfort when receiving or issuing payments with persons in each social group.

First, discussing money and participating in transactions with acquaintances - those with weak social ties - are generally considered comfortable (US1, 10, 15, 19, 20, CN6, 7, 20), involving little social obligation:

For people I don't really know, for strangers, for landlords, I am very comfortable to talk about money. Because I think those are fair transactions.

(US19, female, 21-30)

These transactions only happen when necessary, e.g., paying rent each month. Users generally do not build social connections with these acquaintances. They do not add them to Venmo/WeChat Pay contact lists, or talk about topics beyond the ongoing transaction itself (US1, $2,10,21, \mathrm{CN} 1,2,5,6,7,9,10,11,12,13,15,17)$.

On the other end of the social spectrum are close friends. Participants replied that their transaction frequency with close friends was much higher than with acquaintances. Close friends almost always add each other to their contact lists, in case they want to send/receive money in the future. Although awkwardness arises from time to time when people are unsure of whether money should be involved in dealings with friends (US1, 14, 15, 19, 20, CN6, 10), most participants feel that making transactions with close friends is comfortable and natural (US2, 7, 10, 13, 15, 17, 21, CN1, 2, 8, 9, 11, 13, 19).

Finally, participants mentioned that they feel most uncomfortable when talking about money with normal friends, i.e., day-to-day friends who fill the gap between close friends and mere acquaintances (US2, CN2, 3, 11). They cannot chat naturally as they do with close friends. They cannot directly talk about money as they do with acquaintances, because there are potential negative social consequences. Thus, dealing with normal friends about money requires some initial social lubrication, i.e., small talk.

\section{It feels odd to pay ordinary friends on WeChat since you have to start with conversations while often you just want to make the payment. But it is not the case when paying close friends with whom you often chat. (CN11, female, under 20)}

The results show that users tend to be comfortable with transferring money with close friends or mere acquaintances, while transactions between ordinary friends are perceived to be more socially awkward.

\section{Transactions Affect Social Relationship}

It has been shown that social relationships among users influence their transaction behaviors. It drives us to investigate the effect in the opposite direction; that is, whether and how transaction behaviors affect users' social relationships. We find that digital transactions may not help build new social connections, but it may strengthen existing social ties (H3).

\subsection{Users Rarely Build New Connections}

Both Venmo and WeChat Pay users mentioned that they are not willing to establish new social connections with unfamiliar transaction partners. People usually expect one-time-only transaction with unfamiliar individuals.

In Venmo, even if users have to make multiple transactions with a stranger, they would prefer searching the partner's Venmo username each time rather than keeping it in the friend list (US1, 2, 10, 12, 13, 14, 21). While in WeChat Pay, our respondents indicated that they tend to deliberately avoid exposing their online social network to strangers in a transactional process (CN except 7, 8, 15, 17).

To pay strangers, I will definitely prefer using Alipay or scanning WeChat's payment $Q R$ code 
so that I don't need to follow them in WeChat.

That makes things much easier. (CN3, female, age 21-30)

Even if users have to "friend" someone unfamiliar in WeChat for a payment in certain occasions, they intend to remove the other party soon after the transaction is over. In cases where users need to keep strangers or acquaintances in their contact list for future payments, they seldom chat beyond the transactions with these contacts. In addition, they are likely to separate these connections from their normal social circles, e.g., not sharing moments with them in WeChat (CN except 13, $16,18)$.

These results suggest that $\mathrm{P} 2 \mathrm{P}$ digital wallet may not be suitable for seeking new social relationships that can be extended beyond the financial activities.

\subsection{Transactions Benefit Existing Friendships}

Although the fast transactions enabled by Venmo and WeChat Pay do not leave users much time to socialize during transactions as in [4], it brings in other benefits to existing social ties. They serve to ease interactions around the payment process and enable social-oriented transaction activities.

First, the ease of making transaction mitigates possible social awkwardness and consequent financial risks (US10, CN12, 13, 15, 19). The Chinese participants specifically mentioned that it used to be a pain chasing after friends for bill splitting and sometimes they simply give up (CN12, 19). Now the bill splitting function of WeChat Pay makes the process much easier and more pleasant. The improved commitment to exchange process can generate positive effect towards the other parties involved [41].

Second, the reduced complexity of transactions provides opportunities for social-oriented transactions to take place. For example, in WeChat Pay, the Red Packet feature allows online money gifting in a more casual, lightweight, comfortable manner (CN1, 4, 8, 11, $13,15,19,20)$. It soon became an essential type of social currency that largely boosts social dynamics in the Chinese online communities [39].

In Venmo, users say funny things or even tease each other in the messages associated with transactions, adding playfulness to the whole experience (US9, 14, 17). This is consistent with the previous work [6], which shows seeing funny feeds help people feel more connected to people they care about.

These results are different from the traditional view of money-based exchange, which suggests that money-based exchanges entail issues like objectifying and dehumanizing people, over-concerning wealth

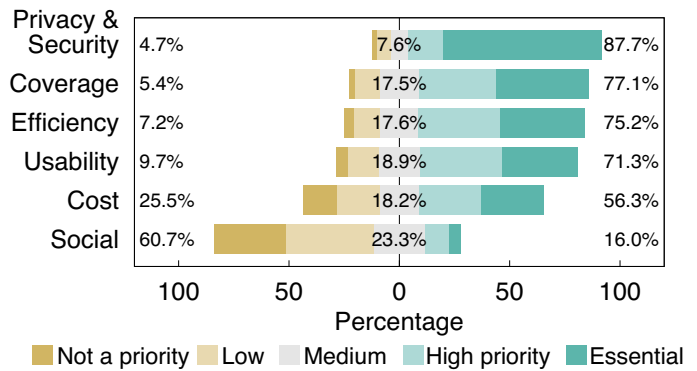

Figure 4. Aspects to be improved to fully embrace digital wallets and eliminate cash (Venmo Users).

and economic benefits, and undermining trust and empathy [42].

\section{Discussion}

\subsection{For Future Social Digital Wallets}

P2P digital payment is a highly competitive market. Many companies have attempted to combine social networks with financial function in their service. However, from users' perspectives, users do not expect the social components to be the most important feature for the digital wallets (Figure 44). Results show that the utility value (i.e., coverage, efficiency, usability and costs for making payment) is the most important considerations. In our interview, participants also suggested that completely removing Venmo's social sharing function would not affect their usage (US except $1,5,16)$. WeChat Pay users mentioned that they would not socialize with contacts added merely for making payments (CN except 13, 16, 18).

Users' expectation of the social feature is to help to facilitate more convenient $\mathrm{P} 2 \mathrm{P}$ payments, rather than "making friends." Our interviewees have responded positively to the ease of locating their friends in Venmo through the Facebook social graph (US1, 6, 8, 10, $14,19,20,21)$ or making payments through WeChat chatting box $(\mathrm{CN} 11,13,18)$. Several WeChat Pay users $(\mathrm{CN} 3,4,17,20)$ stated that paying through the social network service was convenient because they did not need to open another app. This is why they prefer WeChat Pay over other payment systems, such as Alipay and online banks.

\subsection{Limitations}

Our work has several limitations. First, we only study Venmo and WeChat Pay to exemplify the most successful P2P digital wallets. Other online P2P payment systems that support social transactions such 
as Chirpify and Dwolla may also have interesting design features to investigate. Second, recruiting survey/interview participants from Amazon MTurk or social media may entail potential biases [43], as both are nonprobability sampling methods [44]. To alleviate this problem, we incorporate multiple channels to recruit participants in both US and China, to further ensure that our findings are reliable and generalizable. Third, it is inherently difficult to make direct comparisons between WeChat Pay and Venmo, since the two digital wallets have different designs and are introduced in different social contexts. Hence, we mainly provide plausible explanations based on existing theories to any difference observed between the two services in terms of usage and user behaviors.

\section{Conclusion}

In this paper, we conduct a mixed-method study to examine the key factors that contribute to the rapid adoption of P2P digital wallets in the US and China. We particularly focus on the roles and impacts of the built-in social networks in P2P digital wallets. We summarize our key findings and contributions as follows:

- Our survey confirms that the introduction of social features indeed helps to accelerate the initial adoption of Venmo and WeChat Pay. The social feature plays a positive role in mitigating users' security concerns by creating a sense of critical mass, building trusts between users, and facilitating information dissemination and interpersonal persuasion.

- There is a key difference between the US and Chinese users regarding their initial adoption of the digital wallets. Venmo (US) users' adoption decision is more influenced by their close tie(s) (i.e. the "degree effect" ), whereas WeChat Pay users are more likely to be persuaded by the collective opinions and the wide usage in a community (i.e. the "cluster effect").

- Social relationships can affect users' experiences of making peer-to-peer payments. Users are more comfortable transferring money with close friends (strong ties) or acquaintances (weak ties), than with normal friends (those between strong and weak ties).

- Social connections that are built specifically for making payments can hardly transform into real social relationships. Users often deliberately separate these connections from regular social circles. However, friendship of existing ties may benefit from transferring money via $\mathrm{P} 2 \mathrm{P}$ digital wallets, since the experience is more comfortable and fun compared to the conventional payment methods.

\section{Acknowledgments}

The authors would like to thank the anonymous reviewers for their helpful feedback. This project was supported by NSF grants CNS-1527939 and CNS-1705042. Any opinions, findings, and conclusions or recommendations expressed in this material are those of the authors and do not necessarily reflect the views of any funding agencies.

\section{References}

[1] "Send money to friends in messenger." https:// newsroom. fb.com/news/2015/03/send-moneyto-friends-in-messenger/, March 2015.

[2] "Introducing snapcash." https://www.snap.com/enUS/news/post/introducing-snapcash/, November 2014.

[3] H. Wei, "Social networks 'not a way out of trouble' for alipay." http://www. chinadailyasia.com/ business/2017-03/30/content_15593625.html, March 2017.

[4] J. Ferreira, M. Perry, and S. Subramanian, "Spending time with money: From shared values to social connectivity," in Proceedings of the 18th ACM Conference on Computer Supported Cooperative Work $\mathcal{E}$ Social Computing, CSCW '15, (New York, NY, USA), pp. 1222-1234, ACM, 2015.

[5] K.-Y. Lin, Y.-T. Wang, and T. K. Huang, "What drives continued intention for mobile payment?-an expectation cost benefit theory with habit," in Proceedings of the 51st Hawaii International Conference on System Sciences, 2018.

[6] M. Caraway, D. A. Epstein, and S. A. Munson, "Friends don't need receipts: The curious case of social awareness streams in the mobile payment app venmo," PACM on Human-Computer Interaction, vol. 1, no. 1, 2017.

[7] "Paypal annual report 2015." https://investor. paypal-corp.com/annuals-proxies.cfm/, January 2016.

[8] PayPal, "Paypal reports first quarter 2018 results." https://investor.paypal-corp.com/ releasedetail. cfm?ReleaseID $=1065039$, June 2018.

[9] A. Kharpal, "Chinese internet giant tencent launches wechat pay in europe to challenge alibaba's alipay." https://www. cnbc.com/2017/07/10/wechatpay-europe-launch-tencent-to-challengealipay.html, July 2017.

[10] M. Brennan, "Wechat red packets data report of 2018 new year eve." https://chinachannel.co/2018wechat-red-packets-data-report-new-yeareve/, February 2018.

[11] E. M. Rogers, Diffusion of innovations. Simon and Schuster, 2010.

[12] M. Trusov, R. E. Bucklin, and K. Pauwels, "Effects of Word-of-Mouth Versus Traditional Marketing: Findings from an Internet Social Networking Site," Journal of Marketing, vol. 73, no. 5, pp. 90-102, 2009.

[13] A. K. Shah and D. M. Oppenheimer, "Easy does it: The role of fluency in cue weighting," Judgment and Decision Making, vol. 2, no. 6, p. 371, 2007. 
[14] Zsolt Katona, Peter Pal Zubcsek, and M. Sarvary, "Network Effects and Personal Influences: The Diffusion of an Online Social Network," Journal of Marketing Research, vol. 48, no. 3, pp. 425-443, 2011.

[15] D. G. Taylor, T. A. Voelker, and I. Pentina, "Mobile application adoption by young adults: A social network perspective," International Journal of Mobile Marketing, vol. 6, no. 2, pp. 60-70, 2011.

[16] H. Lou, P. Y. Chau, and D. Li, "Understanding individual adoption of instant messaging: An empirical investigation," Journal of the Association for information systems, vol. 6, no. 4, p. 5, 2005.

[17] J. Wang and L. Gu, "Why is wechat pay so popular?," Issues in Information Systems, vol. 18, no. 4, 2017.

[18] X. Zhang, S. Tang, Y. Zhao, G. Wang, H. Zheng, and B. Y. Zhao, "Cold hard e-cash: Friends and vendors in the venmo digital payments system," in Proceedings of International AAAI Conference on Web and Social Media (ICWSM 2017), 2017.

[19] W. T. Yao-Hua Tan, "Toward a generic model of trust for electronic commerce," International Journal of Electronic Commerce, vol. 5, no. 2, pp. 61-74, 2000.

[20] O. Morawczynski and G. Miscione, "Trustworthy mobile transactions: a longitudinal study on m-pesa in kenya," Latusek, $\mathcal{F}$ Gerbasi (Eds.), Trust and Technology, pp. 160-173, 2010.

[21] K. S. Cook and E. Rice, Social Exchange Theory, pp. 53-76. Boston, MA: Springer US, 2006.

[22] P. SHIPTON, "Money and the morality of exchange. jonathan parry and maurice bloch, eds," American Ethnologist, vol. 18, no. 3, pp. 631-631, 1991.

[23] J. Boyd, "In community we trust: Online security communication at ebay," Journal of Computer-Mediated Communication, vol. 7, no. 3, pp. 0-0, 2002.

[24] I. Nitzan and B. Libai, "Social effects on customer retention," Journal of Marketing, vol. 75, no. 6, pp. 24-38, 2011

[25] D. A. Prentice, D. T. Miller, and J. R. Lightdale, "Asymmetries in attachments to groups and to their members: Distinguishing between common-identity and common-bond groups," Personality and Social Psychology Bulletin, vol. 20, no. 5, pp. 484-493, 1994.

[26] G. L. Ciampaglia and D. Taraborelli, "Moodbar: Increasing new user retention in wikipedia through lightweight socialization," in Proceedings of the 18th ACM Conference on Computer Supported Cooperative Work $\mathcal{E}$ Social Computing, CSCW'15, (New York, NY, USA), pp. 734-742, ACM, 2015.

[27] M. Burke, C. Marlow, and T. Lento, "Feed me: Motivating newcomer contribution in social network sites," in Proceedings of the SIGCHI Conference on Human Factors in Computing Systems, CHI '09, (New York, NY, USA), pp. 945-954, ACM, 2009.

[28] X. Gui, Y. Chen, C. Caldeira, D. Xiao, and Y. Chen, "When fitness meets social networks: Investigating fitness tracking and social practices on werun," in Proceedings of the 2017 CHI Conference on Human Factors in Computing Systems, CHI '17, (New York, NY, USA), pp. 1647-1659, ACM, 2017.

[29] G. Simmel and D. Frisby, The philosophy of money. Psychology Press, 2004.

[30] V. A. Zelizer, "The social meaning of money:" special monies"," American journal of sociology, vol. 95, no. 2, pp. 342-377, 1989.
[31] K. D. Vohs, N. L. Mead, and M. R. Goode, "The psychological consequences of money," science, vol. 314, no. 5802, pp. 1154-1156, 2006.

[32] E. S. Poole, C. A. Le Dantec, J. R. Eagan, and W. K. Edwards, "Reflecting on the invisible: Understanding end-user perceptions of ubiquitous computing," in Proceedings of the 10th International Conference on Ubiquitous Computing, UbiComp '08, (New York, NY, USA), pp. 192-201, ACM, 2008.

[33] G. Pritchard, J. Vines, and P. Olivier, "Your money's no good here: The elimination of cash payment on london buses," in Proceedings of the 33rd Annual ACM Conference on Human Factors in Computing Systems, CHI '15, (New York, NY, USA), pp. 907-916, ACM, 2015.

[34] S. Mainwaring, W. March, and B. Maurer, "From meiwaku to tokushita!: Lessons for digital money design from japan," in Proceedings of the SIGCHI Conference on Human Factors in Computing Systems, CHI '08, (New York, NY, USA), pp. 21-24, ACM, 2008.

[35] Y. M. Kow, X. Gui, and W. Cheng, "Special digital monies: The design of alipay and wechat wallet for mobile payment practices in china," in IFIP Conference on Human-Computer Interaction, pp. 136-155, 2017.

[36] V. Bellotti, A. Ambard, D. Turner, C. Gossmann, K. Demkova, and J. M. Carroll, "A muddle of models of motivation for using peer-to-peer economy systems," in Proceedings of the 33rd Annual ACM Conference on Human Factors in Computing Systems, CHI '15, (New York, NY, USA), pp. 1085-1094, ACM, 2015.

[37] C. Teddlie and A. Tashakkori, Foundations of mixed methods research: Integrating quantitative and qualitative approaches in the social and behavioral sciences. Sage, 2009.

[38] R. B. Johnson and A. J. Onwuegbuzie, "Mixed methods research: A research paradigm whose time has come," Educational researcher, vol. 33, no. 7, pp. 14-26, 2004.

[39] Z. Wu and X. Ma, "Money as a social currency to manage group dynamics: Red packet gifting in chinese online communities," in Proceedings of the 2017 CHI Conference Extended Abstracts on Human Factors in Computing Systems, CHI EA '17, (New York, NY, USA), pp. 2240-2247, ACM, 2017.

[40] J. J. Francis, M. Johnston, C. Robertson, L. Glidewell, V. Entwistle, M. P. Eccles, and J. M. Grimshaw, "What is an adequate sample size? operationalising data saturation for theory-based interview studies," Psychology and Health, vol. 25, no. 10, pp. 1229-1245, 2010.

[41] L. D. Molm, N. Takahashi, and G. Peterson, "Risk and trust in social exchange: An experimental test of a classical proposition," American Journal of Sociology, vol. 105, no. 5, pp. 1396-1427, 2000.

[42] J. M. Carroll and V. Bellotti, "Creating value together: The emerging design space of peer-to-peer currency and exchange," in Proceedings of the 18th ACM Conference on Computer Supported Cooperative Work $\mathcal{E}$ Social Computing, CSCW '15, (New York, NY, USA), pp. 1500-1510, ACM, 2015.

[43] R. A. Berk, "An introduction to sample selection bias in sociological data," American Sociological Review, pp. 386-398, 1983.

[44] L. S. Connaway and R. R. Powell, Basic research methods for librarians. ABC-CLIO, 2010. 